\title{
Giant magnetoresistance in a low-temperature GaAs/MnAs nanoscale ferromagnet hybrid structure
}

\author{
P. J. Wellmann, ${ }^{\text {a) }}$ J. M. Garcia, J.-L. Feng, and P. M. Petroff \\ Materials Department, University of California in Santa Barbara, Santa Barbara, California 93106
}

(Received 31 March 1998; accepted for publication 1 October 1998)

\begin{abstract}
We report the observation of a giant magnetoresistance effect in a low-temperature (LT-)GaAs/ MnAs nanoscale ferromagnet hybrid structure. The MnAs nanomagnets are formed by ion implantation of Mn into LT GaAs and subsequent annealing. We have studied the magnetotransport using a vertically biased $p^{+}$-GaAs/LT-GaAs:MnAs/ $p^{+}-$GaAs structure. A negative magnetoresistance $(\Delta \rho / \rho=[\rho(B)-\rho(0)] / \rho(0))$ of up to $-80 \%(B=7 \mathrm{~T})$ is observed at low temperatures $(T<20 \mathrm{~K})$, which changes its sign from negative to positive between $T=15 \mathrm{~K}$ and $T=20 \mathrm{~K}$. The value of the positive magnetoresistance decreases with increasing temperature from $+115 \%(20 \mathrm{~K})$ to $+1.4 \%(300 \mathrm{~K})$. The magnetoresistance variations with $B$ and $T$ are correlated with the nanomagnet spacing in the structure. (C) 1998 American Institute of Physics.
\end{abstract}

[S0003-6951(98)03148-9]

The study of hybrid semiconductor ferromagnet structures based on III-V compounds have gained momentum due to their possible application in new semiconductor devices such as magnetic-field sensors, magneto-optical devices, and memories. Molecular beam epitaxy (MBE) grown diluted magnetic semiconductors like $\operatorname{In}_{1-x} \mathrm{Mn}_{x} \mathrm{As}$ and $\mathrm{Ga}_{1-x} \mathrm{Mn}_{x} \mathrm{As}(x=0-0.09)$ have been the subject of many recent publications ${ }^{1-4}$ with an emphasis on magnetotransport and magneto-optical properties. For large values of $x$ (e.g., $x \leqslant 0.06$ ), MnAs-like clusters may be formed during annealing of $\mathrm{Ga}_{1-x} \mathrm{Mn}_{x} \mathrm{As} .{ }^{5}$ In a different approach, the formation of nanoscale ferromagnetic MnGa and MnAs crystallites using $\mathrm{Mn}^{+}$ion implantation into GaAs or LT GaAs (LT=low temperature) and subsequent annealing has recently been demonstrated. ${ }^{6,7}$ In this letter we present the observation of a giant magnetoresistance effect in the hybrid semiconductor nanomagnet system: LT-GaAs:MnAs using the ion implantation processing technology.

The magnetotransport characteristics have been studied using a vertically biased $p^{+}$-GaAs/LT-GaAs $/ p^{+}-\mathrm{GaAs}$ structure grown by MBE (see the inset of Fig. 1). On top of a semi-insulating (100) GaAs substrate we have deposited 44 periods of a GaAs/AlAs superlattice $(2 \mathrm{~nm} / 2 \mathrm{~nm})$ followed by $500 \mathrm{~nm}$ of $p^{+}$-GaAs $\left(T_{\text {growth }}=630^{\circ} \mathrm{C}, \quad p=5 \times 10^{18}\right.$ $\left.\mathrm{cm}^{-3}\right), 250 \mathrm{~nm}$ of LT GaAs $\left(T_{\text {growth }}=250{ }^{\circ} \mathrm{C}\right.$, undoped $)$, and $50 \mathrm{~nm}$ of $p^{+}$-GaAs $\left(T_{\text {growth }}=350{ }^{\circ} \mathrm{C}, p=5 \times 10^{18} \mathrm{~cm}^{-3}\right)$ with carbon as the $p$ dopant. Manganese ions were implanted with an energy of $180 \mathrm{keV}$ and a dose of $1 \times 10^{16} \mathrm{~cm}^{-2}$. After rapid thermal annealing at $750{ }^{\circ} \mathrm{C}$ in a forming gas atmosphere, nanosize MnAs crystallites are formed in the LT-GaAs layer about $150 \mathrm{~nm}$ below the sample surface. ${ }^{7}$ The samples were capped with a silicon nitride layer (100 $\mathrm{nm})$ to prevent the evaporation of As during heat treatment. Mesa structures of $p^{+}$-GaAs/LT-GaAs $/ p^{+}$-GaAs have been chemically etched and magnetotransport measurements were

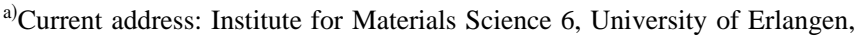
Martens Str. 7, D-91058 Erlangen, Germany. Electronic mail: peter.wellmann@ww.uni-erlangen.de carried out on $2,10,90$, or 100 devices in series. Thermally evaporated $\mathrm{Au} / \mathrm{Zn} / \mathrm{Au}$ layers $(10 \mathrm{~nm} / 10 \mathrm{~nm} / 200 \mathrm{~nm})$ annealed at $420{ }^{\circ} \mathrm{C} \mathrm{(30} \mathrm{s)} \mathrm{were} \mathrm{used} \mathrm{as} \mathrm{Ohmic} \mathrm{contacts} \mathrm{to} \mathrm{the}$ $p^{+}$-GaAs. Magnetotransport measurements were performed in a $\mathrm{He}$ cryostat with a variable temperature insert $(T$ $=2-300 \mathrm{~K})$ and a superconducting magnet $\left(B_{\max }=8 \mathrm{~T}\right)$. We have studied three samples. Samples A and B were Mn implanted and annealed at $750{ }^{\circ} \mathrm{C}$ for 180 and $5 \mathrm{~s}$, respectively. Sample A contains a low density $\left(\approx 10^{9} \mathrm{~cm}^{-2}\right)$ of large MnAs nanomagnets $(d \approx 50 \mathrm{~nm})$ while sample B contains a high density $\left(\approx 10^{10} \mathrm{~cm}^{-2}\right.$ ) of small MnAs nanomagnets $(d \approx 10 \mathrm{~nm})$ imbedded in the LT-GaAs layer. ${ }^{7}$ The mean MnAs crystallite diameter was obtained in transmission electron microscope micrographs. Sample C was a control sample without $\mathrm{Mn}$ implantation, and was annealed at $750{ }^{\circ} \mathrm{C}$ (180 s).

Figure 1 shows the current-voltage characteristics of sample A. In this case 90 devices have been measured in series. At low temperatures $(T=5 \mathrm{~K})$ rectifying behavior dominates, which is typical for $p$-doped/intrinsic/p-doped $(p-i-p)$ structures. The rectifying properties disappear at higher temperature because the thermal activation of holes

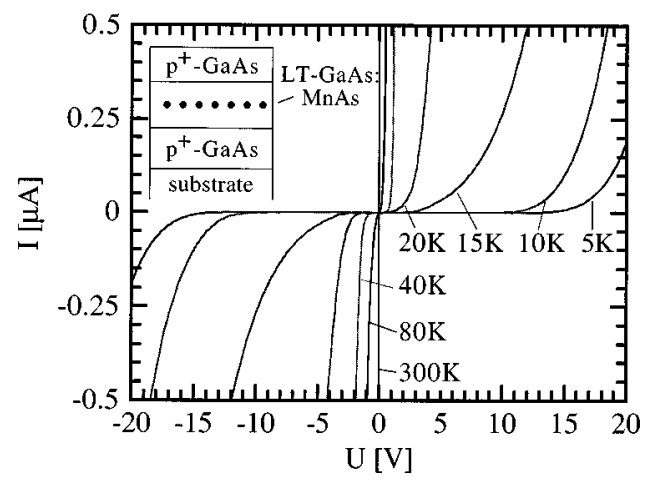

FIG. 1. Temperature dependence of current-voltage characteristics of sample A $\left[[\mathrm{Mn}]=1 \times 10^{16} \mathrm{~cm}^{-2}\right.$, annealed at $\left.750{ }^{\circ} \mathrm{C}(180 \mathrm{~s})\right]$. The inset illustrates the vertical biased $p^{+}$-GaAs/LT-GaAs/ $p^{+}$-GaAs structure containing nanosize MnAs ferromagnets in the LT-GaAs layer. 


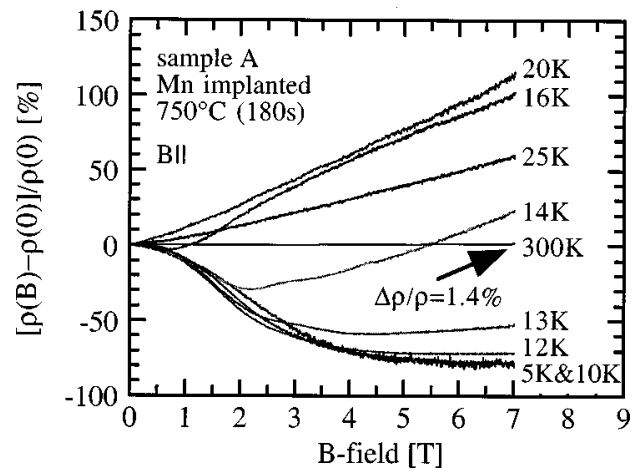

FIG. 2. Temperature dependence of magnetoresistance $\Delta \rho / \rho=[\rho(B)$ $-\rho(0)] / \rho(0)$ of sample A. The $B$ field has been applied parallel to the sample surface $(B \|)$. The applied electrical field was $E=8000 \mathrm{~V} / \mathrm{cm}(T$ $=5-20 \mathrm{~K}), E=6000 \mathrm{~V} / \mathrm{cm}(T=25 \mathrm{~K})$, and $E=100 \mathrm{~V} / \mathrm{cm}(T=300 \mathrm{~K})$.

from the deep acceptor manganese into the valence band of the ion implanted LT-GaAs layer takes over. At room temperature $(300 \mathrm{~K})$ Ohmic behavior dominates.

Figure 2 shows for various temperatures the magnetotransport properties of sample A. The magnetoresistance is defined as $\Delta \rho / \rho=[\rho(B)-\rho(0)] / \rho(0)$ with $\rho$ being the resistivity in $(\Omega \mathrm{cm})$. A giant negative magnetoresistive effect is observed at low temperatures $(T=5 \mathrm{~K}): \Delta \rho / \rho=-3.4 \%$ $(B=0.5 \mathrm{~T})$ and $\Delta \rho / \rho=-80 \% \quad(B=7 \mathrm{~T})$. Between $T$ $=12 \mathrm{~K}$ and $T=16 \mathrm{~K}$ a positive magnetoresistance occurs and reaches its maximum value at $T=20 \mathrm{~K}: \Delta \rho / \rho=+5 \%$ $(B=0.5 \mathrm{~T})$ and $\Delta \rho / \rho=+115 \%(B=7 \mathrm{~T})$. Above $T=20 \mathrm{~K}$ the value of the positive magnetoresistance decreases with increasing temperature to about $1.4 \%$ at $T=300 \mathrm{~K}$. Experiments with the $B$ field applied parallel to the sample surface but in opposite direction reproduce the magnetoresistance data of Fig. 2. Measurements carried out with the $B$ field applied perpendicular to the sample surface instead of parallel also give qualitatively the same results as shown in Fig. 2 (summarized in Table I).

A characteristic feature of the observed magnetoresistance effect is that the sign of $\Delta \rho / \rho$ is correlated with the $E$ field applied across the vertical transport sample for $T$ $\leqslant 20 \mathrm{~K}$ (above $T=20 \mathrm{~K}$ the sign of $\Delta \rho / \rho$ is always positive). The magnetoresistance gradually changes its sign from negative to positive at $T=16 \mathrm{~K}$ (Fig. 3) as the $E$ field decreases from 8600 to $1600 \mathrm{~V} / \mathrm{cm}$. It should be pointed out that due to the diode characteristics of our device (Fig. 1) an initial $E$ field has to be applied to inject charge carriers into the LTGaAs layer. An exponential fit of the $I(V)$ curve revealed an initial $E$ field of $1200 \mathrm{~V} / \mathrm{cm}$ at $T=16 \mathrm{~K}$.

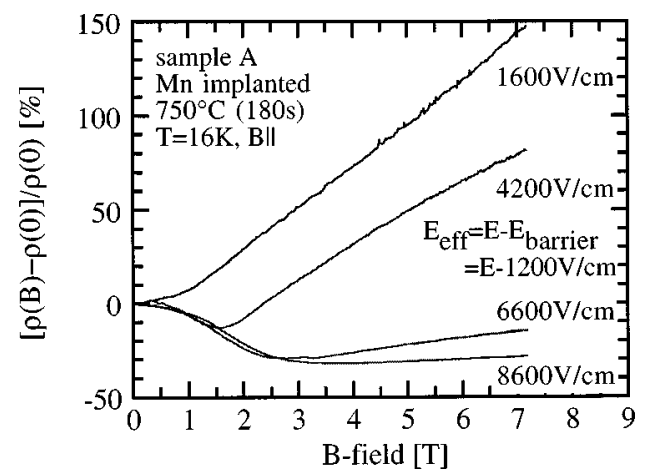

FIG. 3. $E$-field dependence of magnetoresistance $\Delta \rho / \rho=[\rho(B)-\rho(0)] /$ $\rho(0)$ of sample A at $T=16 \mathrm{~K}$. The $B$ field has been applied parallel to the sample surface $(B \|)$.

Sample B, containing $10 \mathrm{~nm}$ MnAs nanomagnets, shows the same behavior as sample A, containing $50 \mathrm{~nm}$ MnAs nanomagnets. However, the observed effects of $\Delta \rho / \rho$ are only in the order of $1 \%$ for sample B versus $100 \%$ for sample A (Table I). The control sample $\mathrm{C}$ with no MnAs nanomagnets imbedded in the LT-GaAs layer shows a small positive magnetoresistance $\Delta \rho / \rho$ in the order of $1 \%$, which is attributed to the well-known geometrical magnetoresistance effect in semiconductors (Table I).

Magnetotransport models proposed for diluted $\mathrm{Mn}_{x} \mathrm{Ga}_{1-x}$ As semiconductors ( $x=3 \%-9 \%$ ) (Refs. 2 and 4) are unlikely to be applicable to our system. These models assume a ferromagnetic ordering of the $\mathrm{Mn}$ spins $\left(\mathrm{Mn}_{\mathrm{Ga}}\right)$ mediated by an antiferromagnetic Mn-hole exchange interaction. In our case the concentration of residual $\mathrm{Mn}$ ions on the lattice site $\left(\mathrm{Mn}_{\mathrm{Ga}}\right)$ is estimated to be $\cong 10^{19} \mathrm{~cm}^{-3}$ and, therefore, more than one order of magnitude less than in the case of diluted semiconductors. Thus, our system is too diluted for a hole mediated exchange interaction of the Mn ions.

Spin-dependent scattering of the charge carriers ${ }^{8}$ at the semiconductor-ferromagnet interface, similar to that found in the granular metallic $\mathrm{Co}-\mathrm{Cu}$ system, ${ }^{9}$ is also not believed to be dominant in our samples. Such an effect would favor sample B containing a high density $\left(10^{10} \mathrm{~cm}^{-2}\right)$ of $10 \mathrm{~nm}$ MnAs nanomagnets, because the spin information has to be carried from one MnAs nanomagnet to the next in order to cause a resistance change due to spin-polarized scattering of the charge carriers at the semiconductor-nanomagnet interface. The data summarized in Table I clearly show that the magnetoresistance effect is significantly larger for sample A containing a low density $\left(10^{9} \mathrm{~cm}^{-2}\right)$ of $50 \mathrm{~nm}$ MnAs nano-

TABLE I. Comparison of magnetoresistance values $\Delta \rho / \rho=[\rho(B)-\rho(0)] / \rho(0)$ for sample A, sample B, and control sample $\mathrm{C}$.

\begin{tabular}{lcccc}
\hline \hline & $\begin{array}{c}\delta \rho / \rho \text { at } 0.5 \mathrm{~T} \\
(5 \mathrm{~K})\end{array}$ & $\begin{array}{c}\Delta \rho / \rho \text { at } 7 \mathrm{~T} \\
(5 \mathrm{~K})\end{array}$ & $\begin{array}{c}\Delta \rho / \rho \text { at } 7 \mathrm{~T} \\
(80 \mathrm{~K})\end{array}$ & $\begin{array}{c}\rho \text { at } B=0 \\
(T<20 \mathrm{~K})\end{array}$ \\
\hline Sample A & $-3.4 \%(B \|)$ & $-80 \%(B \|)$ & $+8 \%(B \|)$ & $140 \mathrm{M} \Omega \mathrm{cm}$ \\
{$\left[\right.$ Mn implanted, $\left.750{ }^{\circ} \mathrm{C}(180 \mathrm{~s})\right]$} & $-10 \%(B \perp)$ & $-25 \%(B \perp)$ & $+12 \%(B \perp)$ & \\
Sample B & $<-0.1 \%(B \|)$ & $-0.4 \%(B \|)$ & $+1.2 \%(B \|)$ & $6.1 \mathrm{M} \Omega \mathrm{cm}$ \\
{$\left[\right.$ Mn implanted, $\left.750{ }^{\circ} \mathrm{C}(5 \mathrm{~s})\right]$} & & & & \\
Sample C & $<+0.1 \%(B \|)$ & $+1.5 \%(B \|)$ & $<+0.1 \%(B \|)$ & $2.9 \mathrm{M} \Omega \mathrm{cm}$ \\
{$\left[\right.$ No Mn, $\left.750{ }^{\circ} \mathrm{C}(180 \mathrm{~s})\right]$} & & & & \\
\hline \hline
\end{tabular}


magnets. Therefore, we conclude that spin-dependent scattering cannot explain the observed magnetoresistance effects.

The comparison of sample A, sample B, and control sample C (Table I) demonstrates that the observed effects are caused by the MnAs nanosize ferromagnets and that the value of $\Delta \rho / \rho$ scales with the size of the nanomagnets. In the simplified approach of a single-domain particle, the $B$ field associated with a nanomagnet is given by a dipole field distribution and is proportional to the magnetic moment $M$ of the MnAs nanomagnet. $M$ is given by $M=N_{\mathrm{Mn}} \mu_{B} g$ where $N_{\mathrm{Mn}}, \mu_{B}$, and $g$ are the number of Mn ions that contribute to the nanomagnet, the Bohr magneton, and the Landé factor, respectively. Therefore, if the magnetoresistance $\Delta \rho / \rho$ scales with the nanomagnet size, it also scales with the magneticfield strength induced by the nanomagnets. Elastic and inelastic scattering of the charge carriers by the nanomagnet dipole field could be related to the observed magnetoresistance effects.

Additional evidence for this scattering mechanism is provided by zero $B$-field electrical measurements. Below $T$ $=20 \mathrm{~K}(B=0 \mathrm{~T})$ the resistivity of samples A, B, and C levels off at different values (Table I). Remarkably, the resistivity is strongly increased in the presence of MnAs nanomagnets. Sample B, containing $10 \mathrm{~nm}$ MnAs nanomagnets shows double the resistivity of control sample $\mathrm{C}$ without MnAs nanomagnets $(6.1 \mathrm{M} \Omega \mathrm{cm}$ vs $2.9 \mathrm{M} \Omega \mathrm{cm})$. Even more striking, the resistivity of sample A containing $50 \mathrm{~nm}$ MnAs nanomagnets is $140 \mathrm{M} \Omega \mathrm{cm}$ vs $2.9 \mathrm{M} \Omega \mathrm{cm}$ for control sample C. We relate this enormous increase in resistivity to the increased path of the charge carriers through the device structure, caused by the scattering by the nanomagnet dipole field.

The underlying physical effects of the observed negative magnetoresistance turn out to be rather complicated. However, we will discuss a simplified semiclassical model which could give further insight. In this model at large applied magnetic fields $(B>2-3 \mathrm{~T})$ and low temperatures ( $T$ $\leqslant 20 \mathrm{~K}$ ) the charge carriers would be forced on cyclotron orbits that are smaller than the average distance of the MnAs nanomagnets. The charge carriers would drift through the LT-GaAs:MnAs layer without interacting with the MnAs nanomagnets, giving rise to a negative magnetoresistance. The cyclotron orbit is given by $R_{c}=v_{h} / \omega_{c}$ and $\omega_{c}=e B / m_{h} v_{h}$ is the hole velocity and $m_{h}$ and $e$ are the electron charge and the effective hole mass $\left(m_{h}=0.5 m_{e} ; m_{e}=\right.$ electron mass $)$, respectively. Due to implantation induced shortcut channels through the substrate we were not able to perform Hall measurements in a lateral device structure in order to determine the hole mobility $\mu_{h}$ and, hence, $v_{h}$. Therefore, we will assume that $v_{h}$ is approximately given by the saturation of the hole drift velocity $v_{d}$. Dalal et al. ${ }^{10}$ have reported that a hole saturation velocity in $p$-doped GaAs of $1.7 \times 10^{7} \mathrm{~cm} / \mathrm{s}$ for $T<100 \mathrm{~K}$. The measured hole drift velocity $v_{d}$ was 1 $\times 10^{7} \mathrm{~cm} / \mathrm{s}$ at an $E$ field of $E=10^{4} \mathrm{~V} / \mathrm{cm}$ and $T=135 \mathrm{~K}$. In the present samples, the implanted and annealed LT GaAs, which contains $10^{9} \mathrm{~cm}^{-2}$ dislocations, we will also assume a similar hole drift velocity at $T \leqslant 20 \mathrm{~K}$. For $v_{h}=1$ $\times 10^{7} \mathrm{~V} / \mathrm{cm}$ and $B=3 \mathrm{~T}$, we find $R_{c}=140 \mathrm{~nm}$, indicating that the cyclotron orbit would be indeed smaller than the average distance of the MnAs nanomagnets of $300 \mathrm{~nm}$. In addition, the $E$-field-dependent magnetotransport data of sample A (Fig. 3) support the existence of a transport regime at large $E$ fields, where the hole mean-free-path length is large enough to force the charge carriers on cyclotron orbits. Increasing the electrical field above $\approx 5000 \mathrm{~V} / \mathrm{cm}$ (at this $E$ field the magnetoresistance changes from a positive to a negative sign) implies an increase of the hole mean-free-path length $l_{h}$ to $>300 \mathrm{~nm}$, which is the average distance of the MnAs nanomagnets. The latter argument, the $E$-field dependent increase of $l_{h}$, holds only if $v_{h}$ is not in saturation at $E<5000 \mathrm{~V} / \mathrm{cm}$.

Above $T=20 \mathrm{~K}$, independent of the applied $E$ field, a positive magnetoresistance is always observed. The temperature dependence of the $I V$ characteristics of sample A (Fig. 1) shows a decrease of the rectifying behavior above $T$ $=20 \mathrm{~K}$, indicating an onset of the thermal activation of holes from the deep acceptor $\mathrm{Mn}$ into the valence band, causing a decrease of the mean-free-path length due charge carrier scattering by ionized $\mathrm{Mn}$ impurities. Above $T=20 \mathrm{~K}$ the positive magnetoresistance can be related to the well-known geometrical magnetoresistance effect. In the absence of a Hall-field, $\Delta \rho / \rho$ is given by $\left(\mu_{h} B\right)^{2}$ with $\mu_{h}=v_{d} E$. With the data given by Dalal et al. ${ }^{10} \quad\left(v_{d}=1 \times 10^{7} \mathrm{~cm} / \mathrm{s}\right.$ at $E$ $\left.=10^{4} \mathrm{~V} / \mathrm{cm}\right), \Delta \rho / \rho$ is approximately $+50 \%$ at $B=7 \mathrm{~T}$. The latter value for $\Delta \rho / \rho$ coincides pretty well with our measurements in Fig. 2: $\Delta \rho / \rho(20 \mathrm{~K})=+100 \%$ and $\Delta \rho / \rho(25 \mathrm{~K})=$ $+50 \%$. However, below $T=20 \mathrm{~K}$ the negative magnetoresistance due to a decreased interaction of the charge carriers with the MnAs nanomagnets is believed to overcompensate the contribution of the positive geometrical magnetoresistance effect.

To clarify whether the semiclassical approach as suggested above is capable of explaining the giant magnetoresistance effect in our samples, a semiclassical calculation for various charge carriers trajectories through the LT-GaAs layer containing the MnAs nanomagnets would be helpful.

This work has been supported by AFOSR/PRET, Center for Non Stochiometric Semiconductors (Grant No. 4962095-1-0394), the Spanish Ministry of Education (J.M.G.), and the Deutsche Forschungsgemeinschaft (P.J.W.).

${ }^{1}$ H. Munekata, H. Ohno, S. von Molnar, A. Segmüller, L. L. Chang, and L. Esaki, Phys. Rev. Lett. 63, 1894 (1989).

${ }^{2}$ H. Ohno, A. Shen, F. Matsakura, A. Oiwa, A. Endo, S. Katsumoto, and Y. Iye, Appl. Phys. Lett. 69, 363 (1996); F. Matsukura, H. Ohno, A. Shen, and Y. Sugawara, Phys. Rev. B 57, R2037 (1998).

${ }^{3}$ T. Hayashi, M. Tanaka, T. Nishinaga, and H. Shimada, J. Appl. Phys. 81, 4865 (1997)

${ }^{4}$ A. Van Esch, L. Van Bockstal, J. De Boeck, G. Verbanck, A. S. van Steenbergen, P. J. Wellmann, B. Grietens, R. Bogarts, F. Herlach, and G. Borghs, Phys. Rev. B 56, 13103 (1997).

${ }^{5}$ J. De Boeck, R. Osterholt, A. Van Esch, H. Bender, C. Bruynseraede, C. Van Hoof, and G. Borghs, Appl. Phys. Lett. 68, 2744 (1996).

${ }^{6}$ J. Shi, J. M. Kikkawa, R. Proksch, T. Schaeffer, D. D. Awschalom, G. Medeiros-Ribeiro, and P. M. Petroff, Nature (London) 377, 707 (1995).

${ }^{7}$ P. J. Wellmann, J. M. Garcia, J.-L. Feng, and P. M. Petroff, Appl. Phys. Lett. 71, 2532 (1997).

${ }^{8}$ H. Akinaga, J. De Boeck, G. Borghs, S. Miyanishi, A. Asamitsu, W. Van Roy, Y. Tomioka, and L. H. Kuo, Appl. Phys. Lett. 72, 3368 (1998).

${ }^{9}$ A. E. Berkowitz, J. R. Mitchell, M. J. Carey, A. P. Young, S. Zhang, F. E. Spada, F. T. Parker, A. Hutten, and G. Thomas, Phys. Rev. Lett. 68, 3745 (1992); J. Q. Xiao, J. S. Jiang, and C. L. Chien, ibid. 68, 3749 (1992).

${ }^{10}$ V. L. Dalal, A. B. Dreeben, and A. Triano, J. Appl. Phys. 42, 2864 (1971).

license or copyright; see http://apl.aip.org/about/rights_and_permissions 